\title{
A B-Learning Model for Training within Electrical Tests Domain
}

\author{
Yasmín Hernández, Miguel Pérez \\ Instituto de Investigaciones Eléctricas, Gerencia de Sistemas Informáticos, \\ Cuernavaca, Mexico \\ \{myhp, mperez\}@iie.org.mx
}

\begin{abstract}
An electrical test performing involves high risk therefore utility companies require high qualified electricians. Traditionally, training on electrical tests has been based on classroom courses; and recently it has been supported by virtual reality systems. These systems have improved training and reduced training time. However the training still depends on courses schedule and instructors, and training systems are not yet adaptive. We propose a model to support adaptive and distance training. The model consists mainly on a representation of the trainees' knowledge and affect. We also proposed an animated pedagogical agent to guide trainees and provide instruction. The agent has facial expressions conveying emotion and empathy to the trainee. This model is intended to be integrated to a virtual reality training system. In this paper, the trainee model and the initial actions of the agent are presented.
\end{abstract}

Keywords: Electrical tests, virtual reality, student model, blended learning.

\section{Introduction}

Training new personnel to perform electrical tests faces some problematic situations such as limited opportunity to practice in a substation, knowledge about electrical tests is not easily available, and training might be costly.

We have developed a virtual reality system (VRS) to support traditional training; trainees still attend classroom courses but they complement learning and practice aided by the VRS. The system has improved training, allows self-training and even both costs and training time have been reduced. However the training still depends on training courses plan and the VRS is not adaptive.

Based on this VRS supporting training, we want to compose a blended learning model. Blended learning is a new term and an innovation in education although the concept has already existed for a long time. It can take many forms and there are several definitions which include roughly the same elements. In a formal education context, blended learning is a formal education program in which a student learns at least in part through online delivery of content and instruction with some element of student control over time, place, path or pace and at least in part at a supervised brick- 
and-mortar location away from home [11]. A more general and perhaps more accepted definition states that blended learning is learning that is facilitated by the effective combination of different modes of delivery, models of teaching and styles of learning, and founded on transparent communication amongst all parties involved with a course [4].

We need a more adaptive training model where the individual state of trainees is considered. We are developing a model and a platform for blended training which adapts to trainee' needs in an intelligent way. Figure 1 shows the road map for training. Currently, we are working in phase 3 and our final aim is to have an intelligent system which supports self-training in an adaptive way (phase 4).

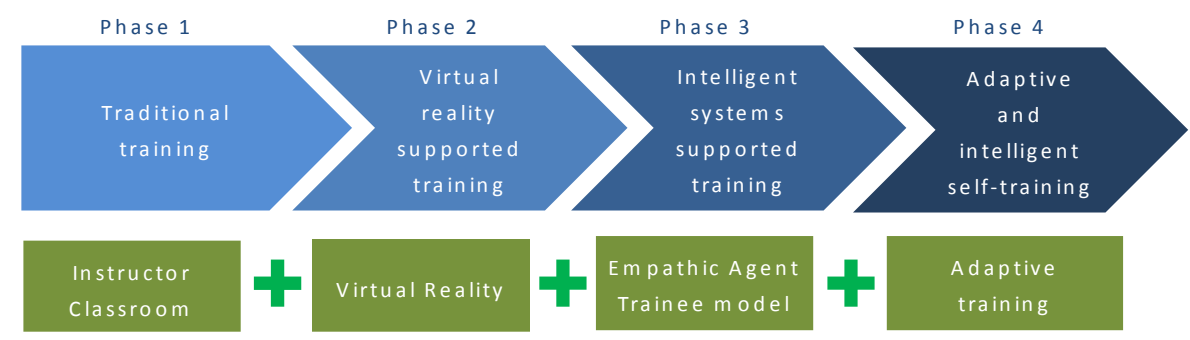

Fig. 1. Training road map for training. The VRS has been implanted successfully, now the empathic agent and trainee model are in development.

This paper presents the work in progress to achieve a system of phase 3 . The rest of the paper is organized as following: Section 2 describes the virtual reality system, Section 3 presents the blended training model. Finally, conclusions and future work are presented in Section 4

\section{3DMapps System}

3DMaPPS is a Virtual Reality Training System created expressly to support electrical tests learning on primary equipment of Distribution Substations [10]. Electrical tests in substations allows foreseeing possible problems, which might end up damaging substation equipment and in turn originating interruptions, which is the ultimate situation expected to be faced by electricity companies. On the other hand, erroneously performed tests might end up in accidents or in equipment damage. Thus, efficient training is mandatory in order to make sure that substation operate in optimum way.

In an overall view of electrical tests, these consist of a sequence of steps where the equipment to be tested has to be bypassed so that is not energized. Then the testing equipment is connected to the primary equipment under test, and outcomes of the tests are recorded. If not other tests is performed, then the testing equipment is disconnected and retired, finally the primary equipment under test in connected again and reestablished. It should be mentioned that in every step safety regulations and measures should be observed [2]. In Figure 2 a transformer being tested is shown. 


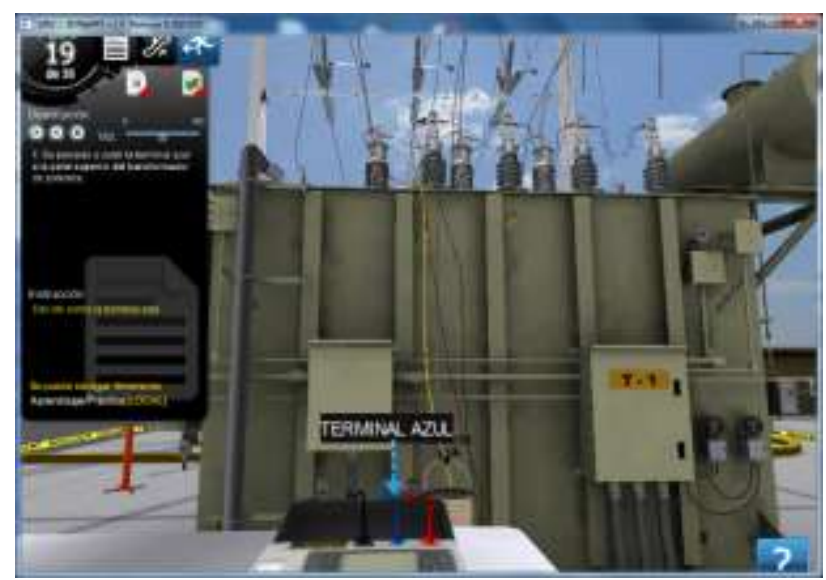

Fig. 2. Testing a transformer. The VR system shows step by step how to perform an electrical test. The trainee is supported by a control panel with several controls and instructions.

3DMaPPS includes 40 electrical tests to different primary equipment such as transformers, interrupters, capacitors bank, and so on. Among the tests, we can mention isolation resistance, power factor, and operation time.

This kind of systems provides advantages derived from 3D representations. For instance, 3DMaPPs contains different catalogs of 3D models of tools, equipment, materials and safety gear; it also includes a $3 \mathrm{D}$ virtual substation. Thus, trainees can visualize $3 \mathrm{D}$ tools and navigate virtually a substation. This allows students to get familiar with all items used when tests are performed. It allows self-learning and provides supports for classroom courses, since it is able to keep records of trainees' progress so that instructors can make even personalized training decision. As in real work, electrical tests are presented as sequences of steps. On each step, explanations are provided and activities are illustrated using 3D animations.

These 3D benefits, make 3DMaPPS a useful complementary training tool, which provides support to the learning process. Using the system, trainees can start learning about electrical tests, even though they have not visited yet a real substation. The system also provides two kinds of evaluation which might be useful when trainees are learning by themselves, namely theoretical questionnaires and practical evaluations. The main objective of the system is to complement and enhance traditional method of training. Nevertheless, the risk of electroshock or damage to equipment, still demands that only human experts can certify trainees, when they consider they are ready to realize electrical tests by themselves.

The system is installed in the 16 distribution areas across the country. This benefits some hundreds of new electricians. The system was developed using OGRE, C\# and 3DSMax. 3DSMax allowed the creations of all 3D models and animations. OGRE, a game engine was used to render scenes and $\mathrm{C \#}$ to develop the interface of the system and organization of presentation of the electrical tests.

Thus far, the system has been helpful as a supporting tool to improve training. Nevertheless other technologies can be integrated to our VR systems, so that they can 
exhibit intelligent and adaptive behaviors which improve them as training tools.

Next section shows the proposal of a model to integrate intelligent and adaptive features to VRS.

\section{Blended Training Model}

The VRS has improved the training process, supporting the traditional training by means of presenting 3D representations of electrical tests and substations. This allows to learn and to practice electrical tests before visiting a substation and perform the real electrical tests. Although 3DMaPPS has shown to be useful in training, it is not adaptable to individual electricians; namely, the system provides instructional content regardless the particular states of trainees. Now we want to provide intelligent support to trainees and trainers.

Currently, we are developing a model and an intelligent system to support traditional training. This new model recognizes the knowledge state of students in order to have more precise information for instructors to grant a certification o to recommend attending other training courses. Moreover, the model includes recognizing the affective state of trainees. Then the instruction is presented in a proper way by an empathic agent. The training model is presented in Figure 3.

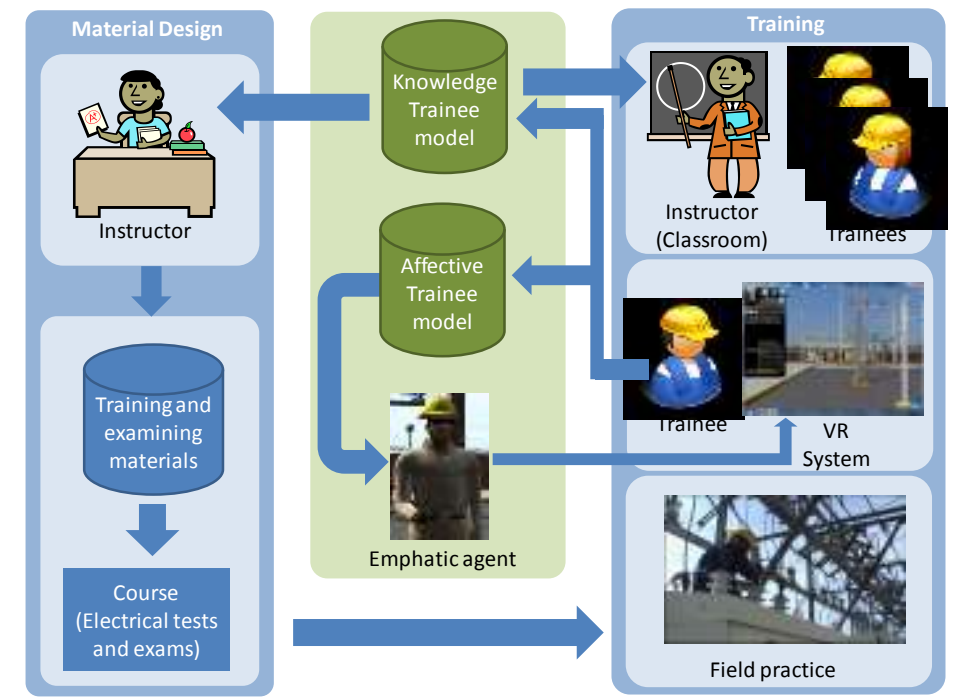

Fig. 3. Blended training model. The model tries to provide instruction in a suitable way by means of an emphatic agent. Also the model provides instructors with knowledge to design material and courses and to adapt the instruction to particular trainees.

The training and examining material were developed and designed by a team of experts. Currently, a course is planned by an instructor. The instructor teaches trainees in classroom and also trainees learn and practice with the VRS as much as they want. 
When trainees have attended the appropriate courses they have to serve as auxiliary electrician in a real substation.

Now we are proposing to model the trainee's knowledge and affect. This trainee model is useful for instructors, who will be able to design new courses and new examining materials, redesign training material and certify trainees. The affect will be used by the emphatic agent to show facial expressions to motivate trainees.

In this third phase, the trainee's knowledge the trainee will not be used to adapt the instruction; since the electrical test have to be thought in a sequence of steps.

\subsection{Pedagogical Trainee Model}

The pedagogical trainee model is a representation of the student's knowledge about electrical tests included in the course. The model is updated when the trainee practices the electrical tests and when he solves theoretical exams. The model consists of a Bayesian network. The Bayesian network is built when the instructor design a course. Fig. 4 shows an example of a network for a course with five electrical tests. In turn each test is composed by a sequence of steps and sub-steps, as shown in Figure 5.

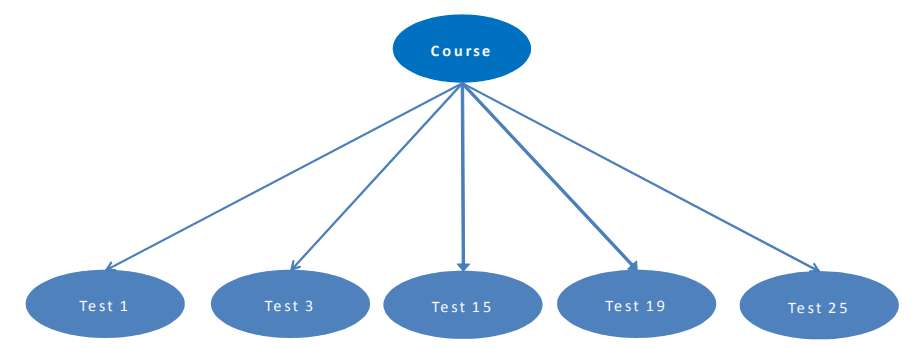

Fig. 4. Bayesian network for a course with five electrical tests. Each test node is another Bayesian network which represents how trainees has performed the electrical tests.

The Bayesian network is composed by a node for each electrical test included in the course and a node for an exam which comprises the items of the theoretical exam also designed by the instructor. In turn, each node of the Bayesian network (see Figure 4) representing a test is a Bayesian network composed by steps and sub-steps. In Figure 5 a test with four steps is shown; but most electrical tests have an average of 40 steps.

Initially, each node representing a sub-step has two possible values: correct and incorrect which correspond to the possible result of performing the sub-step (to execute an instruction). The nodes representing steps have two possible values: learnt and not learnt and their probabilities are conditionally dependent on the probabilities of the substep nodes. Step nodes represent the probability that the trainee has learnt the step. Test nodes also have two values: acquired and not acquired and their probabilities are conditionally dependent on the probabilities of the step nodes.

The theoretical exam is also represented by a Bayesian network composed by a number of items. The causal relationships between items and conditional probabilities for each node will be established when the exam is designed by the instructor. For the time being, we have not defined the complete structure and values of this Bayesian 
network. However we want to model trainee' guesses and slips on the basis of the relationships between the items and the evidence of the answers to questions. Figure 6 shows an exam with 8 items as a preliminary example.

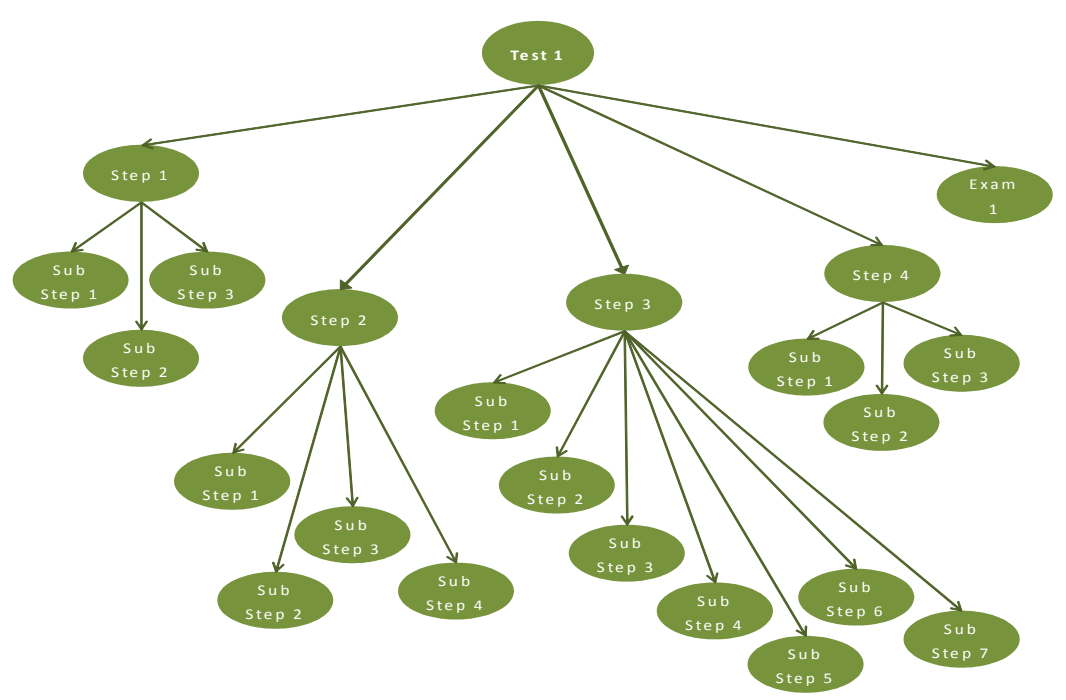

Fig. 5. Bayesian network for an electrical test with four steps. The knowledge about electrical test is impacted by the knowledge of each step and a theoretical exam.

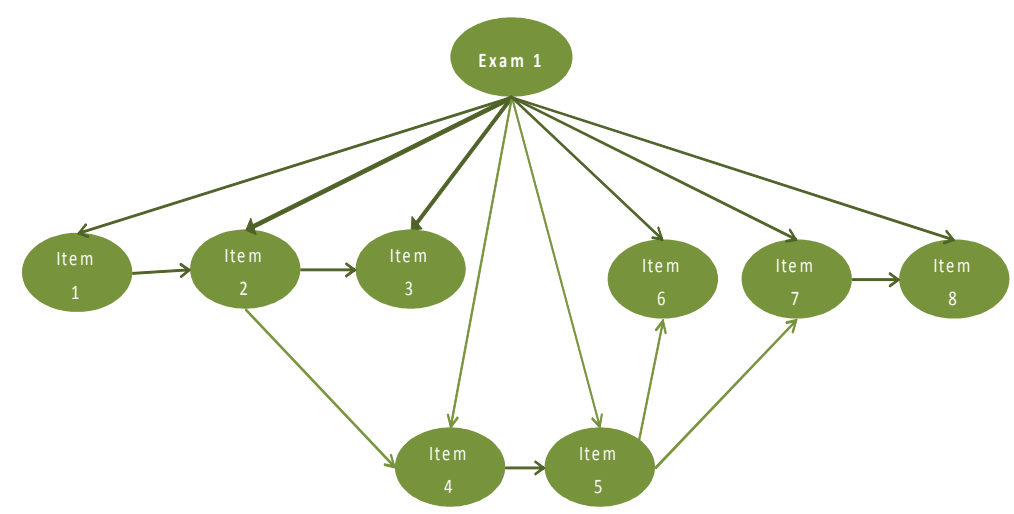

Fig. 6. Bayesian network for an exam including eight items. Initial structure which represents only the relationship between topics of items.

\subsection{Affective Trainee Model}

The affective trainee model uses the OCC model [9] to provide a causal assessment of emotions based on contextual information. The OCC model defines emotional state as the outcome of the cognitive appraisal of the current situation with respect to one's goals. The trainee model consists of a dynamic Bayesian network (DBN) that 
probabilistically relates personality, goals and interaction events with affective states, based on the theory defined by the OCC model. Figure 7 shows a high level representation of the model, where each node in the network is actually a set of nodes in the detailed model. The model is based on the proposal by [3] and in our previous work [5].

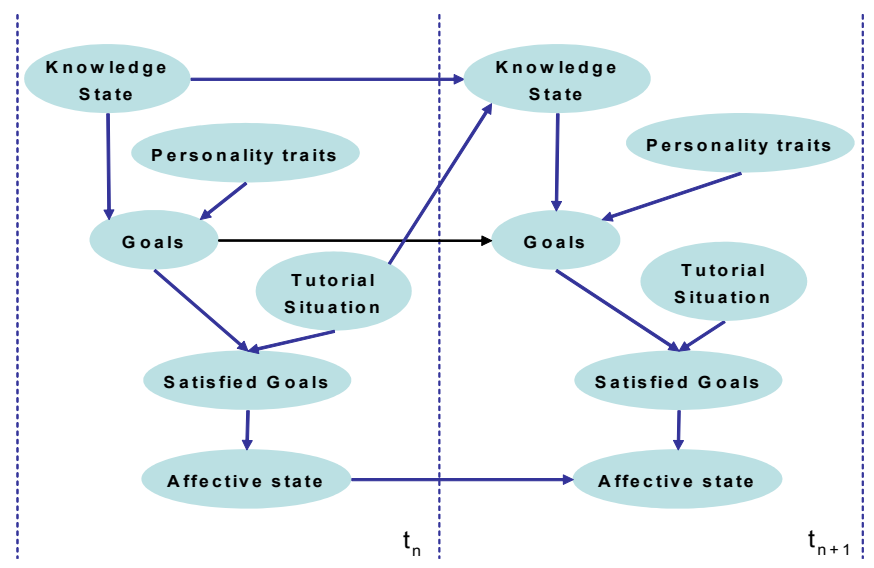

Fig. 7. High level DBN for the affective student model. We include two time slice to represent the dynamic behavior of affect and its impact in the next state.

The DBN models the dynamic nature of emotions. To infer the affective state at $t_{n}$, it considers the student's knowledge, personality, and the tutorial situation at that time, as well as the student affective state at $t_{n-1}$. The tutorial situation is defined based on the results of the trainee actions.

The trainee's appraisal of the current situation given his goal is represented by the relation between the goals and the tutorial situation nodes through the satisfied goals node. The influence of the appraisal process on the trainee's affect is represented by the link between the satisfied goals node and the affective state node. From the complete set of emotions proposed by the OCC model, the affective model only includes six emotions: joy, distress, pride, shame, admiration and reproach.

According to the OCC model, one's goals are fundamental to determine one's affective state, but asking the students to express these goals during training would be too intrusive. Consequently, the goals in our network are inferred from indirect sources of evidence. We use personality traits as a predictor of the student's goals, but we also include the student's knowledge.

\subsection{Animated Agent}

Training activities are presented to trainees through an animated pedagogical agent. These agents represent a major trend to have a more natural human-computer interaction $[1,7]$. Animated pedagogical agents interact face to face with the students through facial expressions, gaze, emotions and deictic gestures; and cohabit with the 
students learning environments. Animated pedagogical agents have a significant impact on training systems as they give the impression that someone is on the other side [12]; thus the trainee perceives a very different behavior from a traditional system and more alike to human behavior. Among the behaviors of an animated pedagogical agent are those typical of intelligent tutoring systems, but there are some particular of these characters, such as demonstrations of complex tasks, observe and assist the trainee to perform their tasks, in addition to guiding trainees in virtual spaces [13].

We are using the characteristics of the operators for developing the agent, such as wearing the uniform and safety helmet, among other features. We believe that by representing the tutor as an electrician, operators will accept the training environment for operators, which is useful for learning. Fig. 8 shows the animated agent.

Empathy is the ability to perceive, understand and experience others' emotions, in other words, step into the shoes of another. This construct has been incorporated in animated agents with the aim to achieve believability, social interaction and user engagement [6].

In this initial phase, the animated agent will deploy the emotions recognized in the student base in the OCC model as described above.

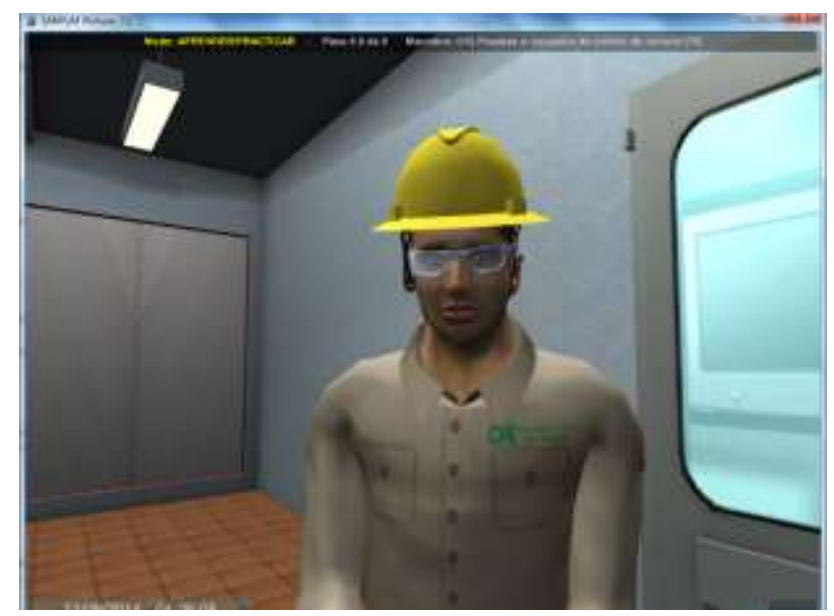

Fig. 8. Animated agent. The animated character wears the uniform and safety gear (Helmet, glasses, cotton uniform, leather boots, gloves and so on) as electricians, trying to convey empathy, besides to the emotional reactions.

\section{Conclusions and Future Work}

There might be instructional domains where learners can self-learn using a system whose instructional content is comprehensive and really well done. In such cases presence of instructor might not be determinant for trainees. Nevertheless, for the systems mentioned here (3DMaPPS), this is not the case. Electrical Test procedures 
involve high risk and also physical activity, which is not provided by a non-immersive VR training system. The point here is that these systems are still limited with respect to the real electrical tests performance, there are physical actions such as claiming up a transformer, removing cables or taking care of safety regulations within specific circumstances, whose expertise cannot be obtained by using the system, but in real work. This is why within this domain involving high risk actions, the systems is not entitled to emit a certificate to enable people to perform electrical tests; this must be responsibility of a human instructor who will have to cover the physical and practical training and verify the skills of the trainees.

Thus, the system is a helpful complementary training tool which can be used to enhance the traditional training but it cannot be used instead of it.

As future work we are planning to show the trainee model to trainee as a selfevaluation tool. Self-assessment is one of the meta-cognitive skills necessary for effective learning. Students need to be able to critically assess their knowledge in order to decide what they need to study [8]. For the time being the open trainee model is used only by instructors.

\section{References}

1. Breese, J. S., Ball, G.: Modeling Emotional State and Personality for Conversational Agents. Technical report. MSR-TR-98-41 (2008)

2. CFE: Handbook of Electrical Test procedures, Comisión Federal de Electricidad. (in Spanish) (2003)

3. Conati, C., Mclaren, H.: Empirically Building and Evaluating a Probabilistic Model of User Affect. User Modeling and User-Adapted Interaction, 19, pp. 267-303 (2009)

4. Heinze, A., Procter, C.: Reflections On The Use Of Blended Learning. University of Salford (2004)

5. Hernández, Y., Sucar, L. E., Arroyo-Figueroa, G.: Affective Modeling for an Intelligent Educational Environment. Intelligent and Adaptive Educational-Learning Systems: Achievements and Trends. Smart Innovation, Systems and Technologies, Springer Book Series, Vol. 17, XII, 532, pp. 3-24 (2013)

6. Hone, K.: Empathic agents to reduce user frustration: The effects of varying agent characteristics. Interacting with Computers, 18(2), pp. 227-245 (2006)

7. Johnson, W. L., Rickel, J. W., Lester, J. C.: Animated Pedagogical Agents: Face-to-Face Interaction in Interactive Learning Environment. International Journal of Artificial Intelligence in Education, 11, pp. 47-78 (2000)

8. Mitrovic, A., Brent M.: Evaluating the Effects of Open Student Models on Learning. In: P. De Bra, P. Brusilovsky (Eds.) AH 2002, LNCS 2347, pp. 296-305 (2002)

9. Ortony, A., Clore, G.L., Collins, A.: The Cognitive Structure of Emotions. Cambridge University Press (1988)

10. Pérez, M.: 3DMapps System. Technical Report, IIE (2014)

11. Staker, H., Horn, M.B.: Classifying K-12 Blended Learning. Innosight Institute (2012)

12. Sagae A., Hobbs J. R., Wertheim S., Agar M., Ho E., Johnson W.L.: Efficient Cultural Models of Verbal Behavior for Communicative Agents. In: IVA 2012, pp. 523-525 (2012) 
Yasmín Hernández and Miguel Pérez

13. Wang N., Johnson W. L., Mayer R. E., Rizzo P., Shaw E., Collins H.: The politeness effect: Pedagogical agents and learning outcomes. International Journal on Human-Computer Studies, vol. 66, no. 2, pp. 98-112 (2008) 